\title{
Superfluidity in the interior-gap states
}

\author{
Shin-Tza Wu and Sungkit Yip \\ Institute of Physics, Academia Sinica, Nankang, Taipei 115, Taiwan
}

(Dated: March 10, 2003)

\begin{abstract}
We investigate superfluidity in the interior-gap states proposed by Liu and Wilczek. At weak coupling, we find the gapless interior-gap state unstable in physically accessible regimes of the parameter space, where the superfluid density is shown to be always negative. We therefore conclude that the spatially-uniform interior-gap phase is extremely unstable unless it is fully gapped; in this case, however, the state is rather similar to conventional BCS states.

PACS numbers: 03.75.Kk, 05.30.Fk, 67.90.+z, 74.20.-z
\end{abstract}

\section{INTRODUCTION}

The advances of techniques in manipulating dilute cold atoms in traps have opened up many fascinating new possibilities to condensed matter physics in recent years. 1] The Bose-Einstein condensation of dilute alkali atoms has provided new Bose-condensed systems previously known only for liquid ${ }^{4} \mathrm{He}$. Experimentalists are now investigating the possibility of realizing the Bardeen-Cooper-Schrieffer (BCS) states in trapped Fermionic atoms. 2, 3, 4] In the simplest case of spin-singlet BCS states, as in ordinary superconductors such as aluminum, the "conventional" Cooper pairs are formed from Fermions with opposite spins. [5] In atom traps, a corresponding realization could be two species of Fermions which pair via the inter-species interaction. The two species of Fermions can be the same kind of atoms (say, ${ }^{6} \mathrm{Li}$ ) in different hyperfine states, or, in the most general case, different Fermionic atoms.

Conventionally, the BCS state is a condensate of Cooper pairs consisting of Fermions with equal mass and opposite spins from states with a single Fermi surface. In considering superconductivity in systems containing ferromagnetically coupled paramagnetic impurities, Fulde and Ferrell, and independently Larkin and Ovchinnikov [] (FFLO), studied Cooper-pairing in systems with mismatched Fermi surfaces. They found a spatially non-uniform phase can be more favored than the BCS states in certain ranges of temperature and Fermisurface mismatches. Recently Liu and Wilczek (LW), motivated by recent developments in atomic physics, studied a system of interacting Fermions consisting of two species of particles with unequal masses and mismatched Fermi surfaces. [6] Assuming pairing only in the vicinity of the smaller Fermi surface, they claimed that at weak coupling there exists a novel superconducting state, which is spatially uniform, could be energetically more favorable than the FFLO state if the coupling strength is above a critical value. This state is characterized by the coexistence of superfluid and normal components at zero temperature that are separated by a momentum gap between the two Fermi surfaces (thus the "interior-gap" state). LW concluded that the critical coupling strength for the interior-gap state could be vanishingly small when the mass difference between the two Fermion species is large.

In this paper, we re-examine the possibility of an interior-gap phase having in mind cold dilute Fermionic atoms in traps. Instead of postulating pairing only around the smaller Fermi surfaces, 6] here we consider a short-range attractive interaction arising from the low energy scattering between Fermions of unlike species. We derive and solve the gap equation, and then further examine the current response of the interior-gap state in the weak coupling limit. Surprisingly, we find the state unstable in all physically accessible regimes unless it is fully gapped.

\section{THE MODEL AND THE GAP EQUATION}

We consider an atom trap which contains two species of Fermions with mass $m_{h}>m_{l}$. We assume that the gas is dilute, with the Fermions interacting via a shortrange interaction (i.e., both the scattering length and the range of the interaction between the two Fermion species are much less than the interparticle distances). This interaction can therefore be modeled by a delta-function like coupling of strength $-g>0$ which ultimately causes pairing between the heavy $(h)$ and the light $(l)$ particles. The system is thus described by the Hamiltonian

$$
H=\sum_{\mathbf{p} \alpha=h, l} \xi_{\mathbf{p} \alpha} a_{\mathbf{p} \alpha}^{\dagger} a_{\mathbf{p} \alpha}+\frac{g}{2} \sum_{\mathbf{p}, \mathbf{p}^{\prime}} a_{\mathbf{p}^{\prime} h}^{\dagger} a_{-\mathbf{p}^{\prime} l}^{\dagger} a_{-\mathbf{p} l} a_{\mathbf{p} h} .
$$

Here $a_{\mathbf{p} \alpha}$ and $a_{\mathbf{p} \alpha}^{\dagger}$ annihilates and creates an $\alpha$ species particle with momentum p. For simplicity, we ignore the trapping potential and take the parabolic dispersions for the particles $\xi_{\mathbf{p} \alpha}=\left(p^{2}-p_{F \alpha}^{2}\right) / 2 m_{\alpha}$ with $p_{F \alpha}$ the Fermi momentum.

Since the gas is dilute, we shall make mean-field approximation and define the space-independent order parameter $\Delta=-g \sum_{\mathbf{p}}\left\langle a_{\mathbf{p} h} a_{-\mathbf{p} l}\right\rangle$ which is chosen to be real. The quasiparticle dispersions are then obtained from the standard procedure, 5 ] yielding

$$
E_{\mathbf{p} h, l}= \pm \frac{\xi_{\mathbf{p} h}-\xi_{\mathbf{p} l}}{2}+\sqrt{\left(\frac{\xi_{\mathbf{p} h}+\xi_{\mathbf{p} l}}{2}\right)^{2}+\Delta^{2}}
$$



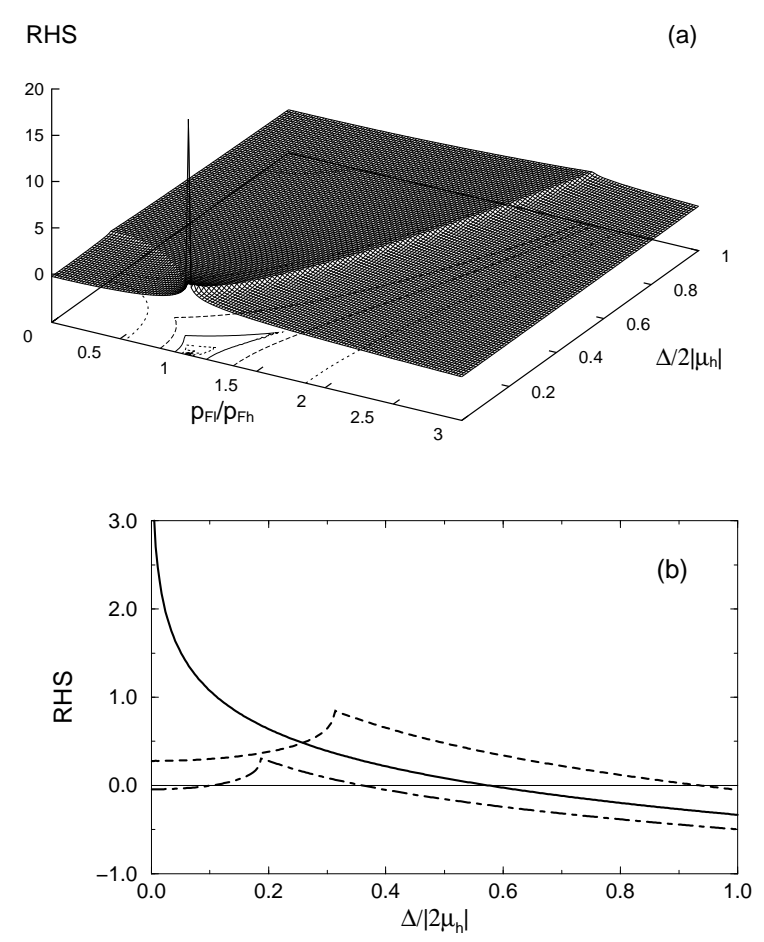

FIG. 1: The right hand side of the gap equation [in units of $\left.2 \pi /\left(p_{F h} m_{r}\right)\right]$ for $m_{h}=m_{l} ;(a)$ shows the three-dimensional plot, and $(b)$ the profiles at $p_{F l} / p_{F h}=0.5$ (dot-dashed line), 1.0 (full line), and 1.5 (dashed line). The ridges in $(a)$ correspond to the critical value $\Delta_{c}$ in Eq. (4) which appear as peaks in $(b)$.

The gap equation is derived from the Hamiltonian upon minimizing the free energy with respect to $\Delta$. However, due to the short-range nature of the pairing interaction, the resulting formula has ultraviolet divergence. To resolve the problem, we eliminate the coupling constant $g$ in favor of the scattering length $a$ for two-particle scattering (between $h$ and $l$ ) in vacuum. The regularized gap equation reads $[8]$

$$
-\frac{m_{r}}{2 \pi a}=\sum_{\mathbf{p}}\left[\frac{1}{E_{h}+E_{l}}\left(1-f\left(E_{h}\right)-f\left(E_{l}\right)\right)-\frac{1}{\xi_{h}^{0}+\xi_{l}^{0}}\right]
$$

where $m_{r}=m_{h} m_{l} /\left(m_{h}+m_{l}\right)$ is the reduced mass, $a$ the $s$-wave scattering length; $f$ is the Fermi function and $\xi_{\alpha}^{0}=p^{2} / 2 m_{\alpha}$ the particle dispersions in vacuum. Here and in below, for brevity, we omit the subscript $\mathbf{p}$ when no confusion would likely arise.

We solve the gap equation at zero temperature by plotting the right hand side (RHS) of Eq. (3) for different values of $m_{l} / m_{h}$ over the parameter space $\left(\bar{p}_{F}, \bar{\Delta}\right)$, where $\bar{p}_{F} \equiv p_{F l} / p_{F h}$ and $\bar{\Delta} \equiv|\Delta| / 2\left|\mu_{h}\right|$ with $\mu_{h}$ the chemical potential of the heavy particles. Since $a<0$ for attractive interactions (without formation of $h-l$ bound states

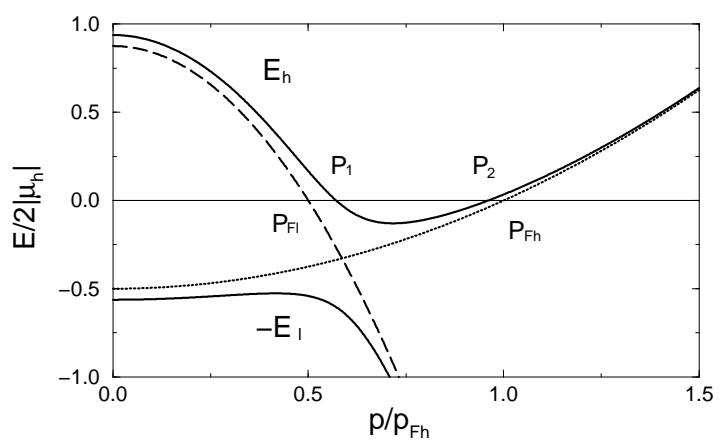

FIG. 2: The quasiparticle dispersions $E_{h}$ and $-E_{l}$ for $m_{h}=$ $7 m_{l}$ and $p_{F h}=2.0 p_{F l}$; the normal-state dispersions $\xi_{h}$ and $-\xi_{l}$ are also shown for the heavy (dotted line) and the light (dashed line) species. Here $\Delta=0.6\left|\mu_{h}\right|<\Delta_{c}=0.99\left|\mu_{h}\right|$. It is seen that $E_{h}$ intersects the $p$-axis at the points $p_{1}$ and $p_{2}$, which correspond to the regions of gapless excitations.

in vacuum), we seek solutions of $\Delta$ such that the RHS is positive. Figure 1 shows typical plots for the RHS of the gap equation with $m_{h}=m_{l}$. The profile at $p_{F l} / p_{F h}=1$ in Fig. प1 $(b)$ thus corresponds to the usual BCS case. In this case the RHS diverges as $\Delta \rightarrow 0$, corresponding to the well-known fact that an infinitesimally small attractive interaction can lead to pairing. However, two differences from the usual BCS states occur here. Firstly, the RHS becomes negative at large $\bar{\Delta}$, indicating no solution for attractive interaction. Secondly, when $p_{F h} \neq p_{F l}$, as dis-

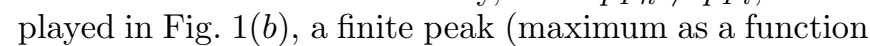
of $\Delta$ ) arises in the RHS of the gap equation at finite $\Delta$. In the three-dimensional plots of Fig. I $(a)$, these peaks appear as ridges over the $\left(\bar{p}_{F}, \bar{\Delta}\right)$ plane. The existence of these peaks (ridges) implies that the system requires a critical coupling strength for solutions to the gap equation when there are Fermi surface mismatches. Another significance of these peaks and ridges is related to the quasiparticle properties of the system, as we shall now explain.

An important feature of the interior-gap state as proposed by LW is the coexistence of the superfluid and the normal components at zero temperature; in other words, there exists gapless excitations. However, as one can check from Eq. (2), this is possible only when the magnitude of the order parameter $|\Delta|$ is smaller than

$$
\Delta_{c}=\frac{\left|p_{F h}^{2}-p_{F l}^{2}\right|}{4 \sqrt{m_{h} m_{l}}} .
$$

When $|\Delta|<\Delta_{c}$, depending on the relative magnitude of $p_{F h}$ and $p_{F l}$, either $E_{h}$ or $E_{l}$ crosses zero at the points (see Fig. [2)

$p_{1,2}=\left(\frac{p_{F h}^{2}+p_{F l}^{2} \mp \sqrt{\left(p_{F h}^{2}-p_{F l}^{2}\right)^{2}-16 m_{h} m_{l} \Delta^{2}}}{2}\right)^{1 / 2}$.

In the event $|\Delta|>\Delta_{c}, E_{h, l}$ both stay positive for all values of $p$. There are then no gapless excitations and the 
interior-gap state has only the superfluid component at zero temperature. The peaks and ridges in the plots for the RHS turn out to locate exactly at the value $|\Delta|=\Delta_{c}$ of Eq. (4). Indeed since $|\Delta|=\Delta_{c}$ draws the boundary between the gapped and the gapless regions over the parameter space, one can expect a qualitative change when plotting the RHS across this line. We will be interested mainly in the gapless regions, namely where $|\Delta|<\Delta_{c}$.

\section{CURRENT RESPONSE}

When $|\Delta|<\Delta_{c}$ the interior-gap state has gapless excitations from the quasiparticle branch whose dispersion crosses zero. In the FFLO ground state, the quasiparticles produce a flow which cancels exactly the current due to the finite-momentum Cooper pairs. This lowers the free energy and stabilizes the FFLO states. 7] In the interior-gap state, the Cooper pairs are stationary in the absence of any superflow. It is therefore of interest to investigate the current response of the interior gap state; in particular, the effects of the quasiparticles in the currentcarrying states.

In the static case the quasiparticle distributions of the interior-gap phase are given by the expression

$$
n_{\mathbf{p} \alpha}^{0}=u_{\mathbf{p}}^{2} f\left(E_{\mathbf{p} \alpha}\right)+v_{\mathbf{p}}^{2} f\left(-E_{\mathbf{p} \bar{\alpha}}\right)
$$

where $\bar{\alpha}$ is the species other than $\alpha$ and the coherence factors are

$$
u_{\mathbf{p}}^{2}=\frac{E_{h}+\xi_{l}}{E_{h}+E_{l}}=\frac{E_{l}+\xi_{h}}{E_{h}+E_{l}}, \quad v_{\mathbf{p}}^{2}=1-u_{\mathbf{p}}^{2} .
$$

In the presence of a small superfluid velocity $\mathbf{w}$, the quasiparticle energies are shifted by $+(\mathbf{p} \cdot \mathbf{w})$ so that the quasiparticle distribution functions of Eq. (6) become

$$
n_{\mathbf{p} \alpha}=u_{\mathbf{p}}^{2} f\left(E_{\mathbf{p} \alpha}+\mathbf{p} \cdot \mathbf{w}\right)+v_{\mathbf{p}}^{2} f\left(-E_{\mathbf{p} \bar{\alpha}}+\mathbf{p} \cdot \mathbf{w}\right) .
$$

The number current can be decomposed as usual into two parts $[5]$

$$
\begin{aligned}
\mathbf{J}_{\alpha}^{p} & =\frac{1}{m_{\alpha}} \sum_{\mathbf{p}} n_{\mathbf{p} \alpha} \mathbf{p} \equiv \rho_{\alpha}^{p} \mathbf{w}, \\
\mathbf{J}_{\alpha}^{d} & =\sum_{\mathbf{p}} n_{\mathbf{p} \alpha} \mathbf{w} \equiv \rho_{\alpha}^{d} \mathbf{w},
\end{aligned}
$$

where the superscripts $p$ and $d$ indicate the paramagnetic and the diamagnetic components; $\rho_{\alpha}^{p, d}$ are the corresponding number densities. In the following we shall examine Eqs. (9) and (10) in the limit of small $\mathbf{w}$ at zero temperature.

Let us consider the case when $p_{F h}>p_{F l}$ and $|\Delta|<\Delta_{c}$. Then, as can be checked from (2), $E_{h}<0$ for $p_{1}<$ $p<p_{2}$ while $E_{l}>0$ always. Therefore the $l$ branch of quasiparticles is always empty, i.e., $f\left(E_{l}\right)=0$ and $f\left(-E_{l}\right)=1$, at zero temperature. In the presence of a small superfluid velocity $\mathbf{w}$, since $E_{l}$ never changes sign, one has

$$
f\left( \pm E_{l}+\mathbf{p} \cdot \mathbf{w}\right)-f\left( \pm E_{l}\right)=0 .
$$

Using the fact that $\mathbf{J}_{\alpha}^{p}=0$ when $\mathbf{w}=0$, we obtain for small superfluid velocity the paramagnetic currents

$$
\begin{aligned}
\mathbf{J}_{\alpha}^{p} & =\frac{1}{m_{\alpha}} \sum_{\mathbf{p}} \mathbf{p}\left(n_{\mathbf{p} \alpha}-n_{\mathbf{p} \alpha}^{0}\right) \\
& \simeq \frac{1}{m_{\alpha}} \sum_{\mathbf{p}} \mathbf{p} \phi_{\alpha}^{2}\left[f\left(\eta_{\alpha} E_{h}+\mathbf{p} \cdot \mathbf{w}\right)-f\left(\eta_{\alpha} E_{h}\right)\right]
\end{aligned}
$$

where for the heavy and the light branches

$$
\phi_{\alpha}=\left\{\begin{array}{l}
u_{\mathbf{p}} \\
v_{\mathbf{p}}
\end{array} \quad \text { and } \quad \eta_{\alpha}=\left\{\begin{array}{l}
+1 \\
-1
\end{array} \quad \text { for } \alpha=\left\{\begin{array}{l}
h . \\
l .
\end{array}\right.\right.\right.
$$

In arriving at the final expression in Eq. (12), we have used (11) for small w.

To leading order in the superfluid velocity, the term in the square brackets in Eq. (12) is proportional to the delta function $\delta\left(E_{h}\right)$, which vanishes except at $p=p_{1}$ and $p=p_{2}$. Replacing the sum by integral, one thus obtains

$$
\rho_{\alpha}^{p}=-\frac{1}{6 \pi^{2}} \frac{1}{m_{\alpha}}\left(p_{1}^{4} D_{1} \phi_{\alpha 1}^{2}+p_{2}^{4} D_{2} \phi_{\alpha 2}^{2}\right),
$$

where $D_{i} \equiv 1 /\left|\partial E_{h} / \partial p\right|_{i}$ and the subscripts $i=1,2$ indicate evaluating at the points $p_{1}, p_{2}$. Note that $\rho_{\alpha}^{p}$ is always negative, which means that the paramagnetic current $\mathbf{J}_{\alpha}^{p}$ always flows in the opposite direction to $\mathbf{w}$.

To leading order in $\mathbf{w}$, the diamagnetic current is simply

$$
\mathbf{J}_{\alpha}^{d}=\sum_{\mathbf{p}} n_{\mathbf{p} \alpha}^{0} \mathbf{w}
$$

which is always in the same direction as w. When $p_{F h}>$ $p_{F l}$ the diamagnetic number-densities can be expressed as

$$
\rho_{h, l}^{d}=\frac{p_{2,1}^{3}}{6 \pi^{2}}+\frac{1}{2 \pi^{2}}\left(-\int_{0}^{p_{1}} \mathrm{~d} p p^{2} u^{2}+\int_{p_{2}}^{\infty} \mathrm{d} p p^{2} v^{2}\right) .
$$

Note that $\rho_{\alpha}^{d}$ is always positive since the diamagnetic current $\mathbf{J}_{\alpha}^{d}$ is always in the same direction as $\mathbf{w}$.

Similar analysis as above can also be done for the case of $p_{F h}<p_{F l}$. As it turns out, we find that the total superfluid number densities $\rho_{\alpha}^{n} \equiv \rho_{\alpha}^{p}+\rho_{\alpha}^{d}$ for the two species are always identical (see Appendix). In other words, despite the seemingly asymmetric properties of the two species, the superflow turns out to be quite conventional - the particles flow together in pairs. The superfluid mass density is thus simply $\rho_{M} \equiv m_{h} \rho_{h}^{n}+m_{l} \rho_{l}^{n}=\left(m_{h}+m_{l}\right) \rho_{h}^{n}$.

As noted earlier, $\rho_{\alpha}^{d}$ is always positive while $\rho_{\alpha}^{p}$ is always negative; as a result, the sign of $\rho_{M}$ is determined from the competition between the paramagnetic 

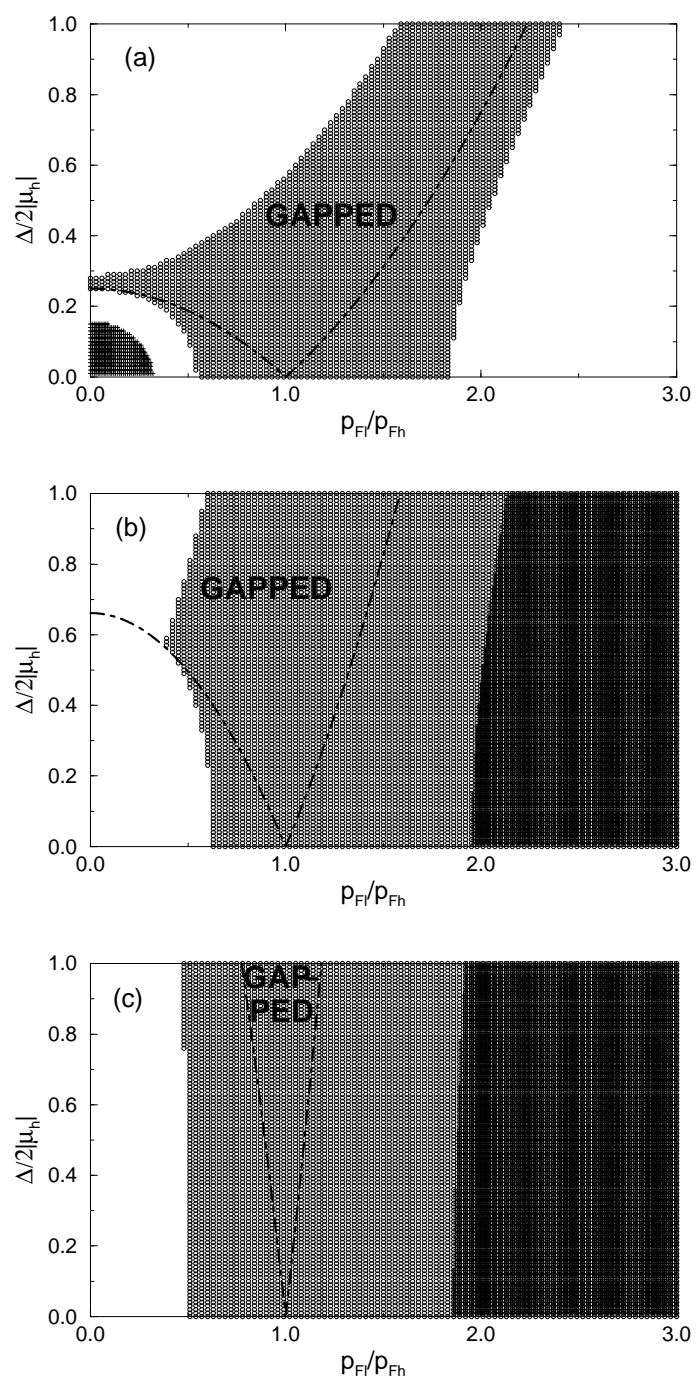

FIG. 3: Summary of our results for the interior-gap states for $m_{l} / m_{h}=(a) 1,(b) 1 / 7$, and $(c) 1 / 100$ over the $\left(\bar{p}_{F}, \bar{\Delta}\right)$ plane. The grey areas are regions where the gap equation has solutions for attractive interactions. The dark areas mark the regions where the superfluid mass density is positive in the gapless regions. The dash-dotted line depicts the critical line determined from Eq. (4), which also corresponds to the ridges in the RHS of the gap equation (see Fig. 1 $(a)$ ).

and the diamagnetic components. Quite unexpectedly, we find the superfluid mass density of the interior-gap phase stays negative in almost all regions of the parameter space. This can also be confirmed analytically for small $\Delta$. A negative superfluid mass density implies that the interior-gap phase is unstable towards a spontaneously generated superfluid velocity or a phase gradient, since the kinetic energy is $\frac{1}{2} \rho_{M} \mathbf{w}^{2}<0$. Our results for $m_{l} / m_{h}=1,1 / 7$, and $1 / 100$ are shown in Fig. [3 From Fig. B] $(a)$ one observes that for $m_{h}=m_{l}$ the regions where $\rho_{M}>0$ and the regions where the gap equation has solutions are completely disjoint to each other in the

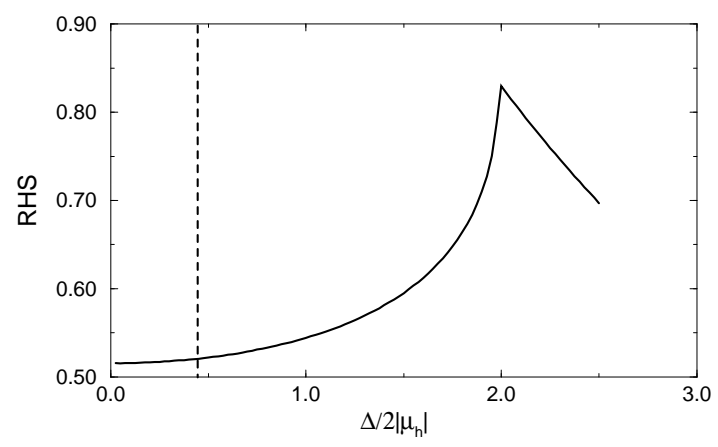

FIG. 4: The RHS of the gap equation [in units of $2 \pi /\left(p_{F l} m_{r}\right)$ ] for $m_{h}=7 m_{l}$ at $p_{F l}=2 p_{F h}$. The dashed line indicates the value of $\bar{\Delta}$ below which the superfluid mass density is positive (cf. Fig. 3 $(b)$ ). To the right of the peak, the state becomes fully gapped, so that the mass density is again positive trivially.

gapless region. Namely for $m_{h}=m_{l}$, except in the fully gapped region, there is no where in the parameter space $\left(\bar{p}_{F}, \bar{\Delta}\right)$ the interior-gap state being stable. This is consistent with the FFLO results. 7] For larger mass ratio at $m_{h}=7 m_{l}$, as shown in Fig. B $3(b)$, there are regions in the gapless area where $\rho_{M}$ stays positive and the gap equation has solutions. To estimate the coupling strength required, we plot in Fig. 4 the profile of the RHS of the gap equation at $p_{F l}=2 p_{F h}$. Disregarding the fact that there are two solutions to the gap equation in this case (see next Section for a discussion), we find the coupling strength necessary for $\rho_{M}>0$ around $\left|p_{F l} a\right| \sim 1.9$. At such strong couplings, however, the validity of the present mean-field calculation is doubtful. For even larger mass ratio at $m_{h}=100 m_{l}$, the situation stays much the same (see Fig. 31 (c)); at $p_{F l}=2 p_{F h}$ we find $\left|p_{F l} a\right| \sim 0.7$ for $\rho_{M}$ to be positive. Even though the coupling strength does go down with increasing mass ratio, it is unrealistic to consider even higher values of $m_{h} / m_{l}$. Note also that the regions of $\rho_{M}>0$ occurs for $p_{F l}>p_{F h}$, instead of $p_{F l}<p_{F h}$ as suggested by LW. [6]

From the sign of the superfluid density, our results show that in the interior-gap phase the paramagnetic component overwhelms the diamagnetic component whenever there are gapless excitations. It is only when the quasiparticles are gapped out the number density in the interior-gap state would stay positive at weak couplings.

\section{SUMMARY AND DISCUSSION}

Within the range of validity of our calculation, we find the interior-gap phase unstable in physically accessible regions of the parameter space; the superfluid mass density is always negative at weak couplings, rendering a uniform phase unstable. Therefore, starting from the normal state, upon increasing coupling strength the sys- 
tem considered here would/may first enter an inhomogeneous FFLO state which persists until the onset of a fully gapped "interior-gap" state; a uniform gapless interiorgap state has no room in the intervening regimes. Indeed this may have been expected from continuity: As was noticed, due to the existence of the peaks and ridges in the RHS, there can be two solutions to the gap equation (see Fig. (1). Since at $p_{F h}=p_{F l}$ the only solution is always in the gapped region, from continuity one expects the stable solutions when $p_{F h} \neq p_{F l}$ to be those above the $\mathrm{V}$ shape boundaries in Fig. 3] In other words, continuity implies that it is always the gapful solutions that are stable. This includes the large $\left|p_{F l} a\right|$ regimes discussed near the end of the last section. For this fully gapped phase, there are no quasiparticles at zero temperature and the number densities of the $h$ and the $l$ branches are always equal. [10] This state thus behaves just like an ordinary BCS state.

This research was supported by NSC of Taiwan under grant number NSC 91-2112-M-001-063.

\section{Appendix}

In this appendix we show that the superfluid number densities for the heavy and the light species are identical at small superfluid velocities.

Let us consider first the case of $p_{F h}>p_{F l}$. We note first that from Eqs. (2) and (7)

$$
\frac{\partial E_{h}}{\partial p}=p\left(\frac{u^{2}}{m_{h}}-\frac{v^{2}}{m_{l}}\right) .
$$

Substituting the above expression into Eq. (14), we ob- tain the difference between the paramagnetic number densities of the two species $[9]$

$$
\begin{aligned}
\rho_{h}^{p}-\rho_{l}^{p}=-\frac{1}{6 \pi^{2}} \quad & {\left[p_{1}^{3} \operatorname{sgn}\left(\frac{u_{1}^{2}}{m_{h}}-\frac{v_{1}^{2}}{m_{l}}\right)\right.} \\
& \left.+p_{2}^{3} \operatorname{sgn}\left(\frac{u_{2}^{2}}{m_{h}}-\frac{v_{2}^{2}}{m_{l}}\right)\right] .
\end{aligned}
$$

Noting that $E_{h}\left(p_{1,2}\right)=0$, one can derive from (17)

$$
\frac{u_{1}^{2}}{v_{1}^{2}}<\frac{m_{h}}{m_{l}}, \quad \frac{u_{2}^{2}}{v_{2}^{2}}>\frac{m_{h}}{m_{l}} .
$$

Therefore (A2) becomes

$$
\rho_{h}^{p}-\rho_{l}^{p}=-\frac{1}{6 \pi^{2}}\left(p_{2}^{3}-p_{1}^{3}\right) .
$$

For the diamagnetic number densities, at small superfluid velocity the difference between the two species is simply that of their quasiparticle occupation numbers. As one can find trivially from Eq. (16)

$$
\rho_{h}^{d}-\rho_{l}^{d}=+\frac{1}{6 \pi^{2}}\left(p_{2}^{3}-p_{1}^{3}\right) .
$$

Combining Eqs. (A 4) and (A 5), we conclude that $\rho_{h}^{n}-$ $\rho_{l}^{n}=0$, namely that the superfluid number densities of the two species are identical.

For $p_{F h}<p_{F l}$ similar calculations as above lead to

$$
\begin{aligned}
\rho_{h}^{p}-\rho_{l}^{p} & =+\frac{1}{6 \pi^{2}}\left(p_{2}^{3}-p_{1}^{3}\right), \\
\rho_{h}^{d}-\rho_{l}^{d} & =-\frac{1}{6 \pi^{2}}\left(p_{2}^{3}-p_{1}^{3}\right) .
\end{aligned}
$$

Therefore, again, one finds $\rho_{h}^{n}=\rho_{l}^{n}$ in this case.
[1] See, eg., E. A. Cornell and C. E. Wieman, Rev. Mod. Phys. 74, 875 (2002), C. J. Pethick and H. Smith, BoseEinstein condensation in dilute gases (Cambridge University Press, Cambridge, 2002), and review articles in Nature 416, 206 (2002).

[2] B. DeMarco and D. S. Jin, Science 285, 1703 (1999); B. DeMarco, S. B. Papp, and D. S. Jin, Phys. Rev. Lett. 86, 5409 (2001).

[3] A. G. Truscott, K. E. Strecker, W. I. McAlexander, G. B. Partridge, and R. G. Hulet, Science 291, 2570 (2000); F. Schreck, L. Khaykovich, K. L. Corwin, G. Ferrari, T. Bourdel, J. Cubizolles, and C. Salomon, Phys. Rev. Lett. 87, 080403 (2001).

[4] Z. Hadzibabic, C. A. Stan, K. Dieckmann, S. Gupta, M. W. Zwierlein, A. Görlitz, and W. Ketterle, Phys. Rev. Lett. 88, 160401 (2002); G. Roati, F. Riboli, G. Modugno, and M. Inguscio, Phys. Rev. Lett. 89, 150403 (2002).

[5] See, eg., M. Tinkham, Introduction to superconductivity, 2nd ed. (McGraw-Hill, New York, 1996).

[6] W. V. Liu and F. Wilczek, Phys. Rev. Lett. 90, 047002 (2003).

[7] P. Fulde and R. A. Ferrell, Phys. Rev. 135, A550 (1964); A. I. Larkin and Yu. N. Ovchinnikov, Zh. Eksp. Teor. Fiz. 47, 1136 (1964) [translation: Sov. Phys. JETP 20, $762(1965)]$.

[8] C. A. R. Sá de Melo, M. Randeria, and Jan R. Engelbrecht, Phys. Rev. Lett. 71, 3202 (1993).

[9] One may notice that when $u_{i}^{2} / m_{h}=v_{i}^{2} / m_{l}$, Eq. A1 vanishes and the expression (14) becomes singular. However, this takes place only when the dispersion curve is tangent to the energy zero, i.e. when $|\Delta|=\Delta_{c}$. Since we are interested in the case of $|\Delta|<\Delta_{c}$, this shall not concern us.

[10] This means that there must be phase separation in this fully gapped phase, since in general the $h$ and the $l$ particles can have unequal numbers in the normal state. 